\title{
On a Harnack inequality for monlinear parabolic equations
}

\author{
By \\ Mitunobu Kurihara

\section{§1. Introduction}

We have been interested in studying the local behaviors of (weak) solutions of partial differential equations with discontinuous coefficients. In particular in this paper we wish to obtain a Harnack inequality for solutions of quasi-linear partial differential equations of second order parabolic type and to derive interior estimates, one of which is uniform Hölder continuity for all bounded solutions of these equations. The most remarkable result which ensures a Harnack inequality and uniform Hölder continuity of solutions of partial differential equations with discontinuous coefficients was established by J. Moser [5] in 1964 for linear uniformly parabolic equations which consist of terms of second order derivatives. He extended his own method which had been introduced by himself [2] [3] for uniformly elliptic equations in the corresponding form. This method of J. Moser's was applied to derive the Hölder continuity of solutions of linear elliptic and parabolic equations by С. Н. Кружков [9] [10] [11] and also applied to derive a Harnack inequality and Hölder continuity of solutions of quasi-linear partial differential equations of elliptic type by J. Serrin [13].

In this paper we are concerned with certain class of real quasilinear partial differential equations of second order parabolic type;

$$
\operatorname{div} A\left(x, t, u, u_{x}\right)-u_{t}=B\left(x, t, u, u_{x}\right) .
$$

Here, $A(x, t, u, p)$ is a given vector valued function of variables $x=\left(x_{1}, \cdots, x_{n}\right), t, u$ and $p=\left(p_{1}, \cdots, p_{n}\right) . B(x, t, u, p)$ is a scalar valued function of the same variables. $u_{x}$ and $u_{t}$ denote the gradient of the 
function $u=u(x, t)$ with respect to $x$ and $t$ respectively, that is,

$$
u_{x}=\left(\frac{\partial u}{\partial x_{1}}, \cdots, \frac{\partial u}{\partial x_{n}}\right) \text { and } u_{t}=\frac{\partial u}{\partial t} .
$$

We assume that $A(x, t, u, p)$ and $B(x, t, u, p)$ are defined for all points $(x, t)$ in a "rectangle" domain $R$ in $(n+1)$-dimensional Euclidean space and for all values of $u$ and $p$. We denote by $R$ the rectangle domain;

$$
\left\{(x, t) ; x=\left(x_{1}, \cdots, x_{n}\right),\left|x_{i}\right|<k r, i=1, \cdots, n, 0<t<h r^{2} .\right\},
$$

where $k, h$ and $r$ are positive constants. If we denote by $Q_{\rho}$ the subset in $n$-dimensional Euclidean space;

$$
\left\{x ; x=\left(x_{1}, \cdots, x_{n}\right),\left|x_{i}\right|<\rho, i=1, \cdots, n\right\},
$$

then we can express $R$ in the following;

$$
R=Q_{k r} \times\left(0, h r^{2}\right) .
$$

Furthermore we assume that the functions $A(x, t, u, p)$ and $B(x$, $t, u, p)$ are to satisfy the inequalities of the form;

$$
\begin{aligned}
& |A(x, t, u, p)| \leqq a|p|+c|u|+e \\
& |B(x, t, u, p)| \leqq b|p|+d|u|+f \\
& p \cdot A(x, t, u, p) \geqq a^{-1}|p|^{2}-d|u|^{2}-g
\end{aligned}
$$

where $a$ is a positive constant and the coefficients $b$ through $g$ are nonnegative measurable functions of $(x, t)$ defined in the domain $R$, all the following values of which are finite.

$$
\begin{aligned}
& \max _{0<t<h r^{2}}\left(\int_{Q_{k r}} b^{\frac{n}{1-\varepsilon}} d x\right)^{\frac{1-\varepsilon}{n}}, \max _{0<t<h r^{2}}\left(\int_{Q_{k r}} c^{\frac{n}{1-\varepsilon}} d x\right)^{\frac{1-\varepsilon}{n}}, \\
& \max _{0<t<h r^{2}}\left(\int_{Q_{k r}} d^{\frac{2}{n-\varepsilon}} d x\right)^{\frac{2-\varepsilon}{n}}, \max _{0<t<h r^{2}}\left(\int_{Q_{k r}} e^{\frac{n}{1-\varepsilon}} d x\right)^{\frac{1-\varepsilon}{n}}, \\
& \max _{0<t<h r^{2}}\left(\int_{Q_{k r}} f^{\frac{n}{2-\varepsilon}} d x\right)^{\frac{2-\varepsilon}{n}}, \max _{0<t<h r^{2}}\left(\int_{Q_{k r}} g^{\frac{n}{2-\varepsilon}} d x\right)^{\frac{2-\varepsilon}{n}},
\end{aligned}
$$

for $0<\varepsilon<1$.

Throughout this paper we denote by $\|h\|_{s}^{(R)}$ the following norm of a measurable function $h(x, t)$ in $R=Q_{k r} \times\left(0, h r^{2}\right)$; 


$$
\|h\|_{s}^{(R)}=\max _{0<t<h r^{2}}\left(\int_{Q_{k r}}|h(x, t)|^{s} d x\right)^{1 / s}
$$

Here, max (min) stands for essential maximum (essential minimum). Similarly nonnegativity of a function $h$ is used when the set where $h<0$ is of measure zero. $|A(x, t, u, p)|$ and $|p|$ in (1.2) should be understood as the Euclidean length of $n$-dimensional vectors $A(x, t, u, p)$ and $p$ respectively.

In $\S 2$ we shall assert our main theorem (Theorem 1 ), that is, a Harnack inequality for solutions of the equations (1.1) and derive two corollaries as the consequences of Theorem 1 . In $\S 3$ and $\S 4$ we shall derive various estimates of solutions of the equations (1.1) in order to prove our main theorem. $\$ 5$ contains the proof of Theorem 1 .

The author would like to express his gratitude to Professor M. Hukuhara for helpful advices.

\section{$\S 2$. Our main results}

Definition 1. A solution of the equation (1.1) in the domain $R$ is a function $u=u(x, t)$ for which the first derivatives $u_{x}, u_{t}$ and $u$ itself are square integrable in $R$ and which satisfies

$$
\iint_{R}\left[\phi u_{t}+\phi_{x} \cdot A\left(x, t, u, u_{x}\right)+\phi B\left(x, t, u, u_{x}\right)\right] d x d t=0
$$

for every infinitely often differentiable function $\phi(x, t)$ which for every fixed $t$ has compact support as a function of $x$.

Definition 2. A subsolution (supersolution) of the equation (1.1) in the domain $R$ is a function $u=u(x, t)$ for which the first derivatives $u_{x}, u_{t}$ and $u$ itself are square integrable in $R$ and which satisfies

$$
\iint_{R}\left[\phi \cdot u_{t}+\phi_{x} \cdot A\left(x, t, u, u_{x}\right)+\phi \cdot B\left(x, t, u, u_{x}\right)\right] d x d t \leqq(\geqq) 0
$$

for every nonnegative infinitely often differentiable function $\phi(x, t)$ which for every fixed $t$ has compact support as a function of $x$. (See [5)].

To formulate our main results, we consider in the domain $R$ three subdomains;

$$
R^{\prime}=\left\{(x, t) ;\left|x_{i}\right|<k \rho, i=1, \cdots, n, 0<t<h \rho^{2} .\right\}
$$




$$
\begin{aligned}
& R^{+}=\left\{(x, t) ; \quad\left|x_{i}\right|<k_{1} \rho, i=1, \cdots, n, h^{+} \rho^{2}<t<h \rho^{2} .\right\} \\
& R^{-}=\left\{(x, t) ; \quad\left|x_{i}\right|<k_{1} \rho, i=1, \cdots, n, h_{1}^{-} \rho^{2}<t<h_{2}^{-} \rho^{2} .\right\}
\end{aligned}
$$

where, $\quad 0<\rho \leqq r, 0<k_{1}<k, 0<h_{1}^{-}<h_{2}^{-}<h^{+}<h$, and we suppose that

$$
(k r)^{\eta} \cdot\|b\|_{\frac{n}{1-\varepsilon}}^{(R)},(k r)^{\kappa} \cdot\|c\|_{\frac{n}{1-\varepsilon}}^{(R)},(k r)^{\kappa} \cdot\|d\|_{\frac{n}{2-\varepsilon}}^{(R)} \leqq \lambda,
$$

for some constant $\lambda$, where

$$
\eta=\left\{\begin{array}{ccc}
\varepsilon ; & n \geqq 3 \\
\frac{2+\varepsilon}{3} ; & n=1 \\
\frac{1+2 \varepsilon}{3} ; & n=2
\end{array} \quad \kappa=\left\{\begin{array}{cc}
\varepsilon ; & n \geqq 3 \\
\frac{2+2 \varepsilon}{3} ; & n=1 \\
\frac{4+\varepsilon}{3} ; & n=2 .
\end{array}\right.\right.
$$

Theorem 1. Suppose that $u=u(x, t)$ is a solution of the equation (1.1) which is nonnegative in $R^{\prime} \subset R$, then

$$
\max _{R^{-}} u \leqq r\left\{\min _{R^{+}} u+L(\rho)\right\},
$$

where $\gamma$ is a positive constant which depends on $n, a, \lambda, \varepsilon, k_{1} h^{+}, h_{1}^{-}, h_{2}^{-}$ alone, and

$$
L(\rho)=\rho^{\eta}\|e\|_{\frac{n}{1-\varepsilon}}^{(R)}+\rho^{\kappa}\|f\|_{\frac{n}{2-\varepsilon}}^{(R)}+\left(\rho^{k}\|g\|_{\frac{n}{2-\varepsilon}}^{(R)}\right)^{1 / 2} .
$$

Using Theorem 1, we can derive two corollaries which ensure certain interior estimates of solutions. In this case we can generalise the form of the domain and denote by $\Omega$ a domain $\Omega_{0} \times(0, T)$, where $\Omega_{0}$ is an open domain in the $n$-dimensional Euclidean space.

We introduce for a point $(y, s)$ a one-sided $\delta$-neighborhood by the inequalities;

$$
\left|x_{i}-y_{i}\right|<\delta, \quad i=1, \cdots, n,-\delta^{2}<t-s<0 \text {. }
$$

Let $\Omega_{\delta}$ be a subdomain of $\Omega$ such that with $(x, t) \in \Omega$ also a $\delta$-neighborhood in $\Omega$. Furthermore we assume instead of (2.3) and (2.5) that

$$
\begin{aligned}
& \|b\|_{\frac{n}{1-\varepsilon},(\rho)}^{(\rho)},\|c\|_{\frac{n}{1-\varepsilon}}^{(\rho)},\|d\|_{\frac{n}{2-\varepsilon}}^{(\rho)} \leqq \lambda \\
& \|e\|_{\frac{n}{1-\varepsilon}}^{(\rho)},\|f\|_{\frac{n}{2-\varepsilon}}^{(\rho)},\|g\|_{\frac{n}{2-\varepsilon}}^{(\rho)} \leqq \mu .
\end{aligned}
$$

Corollary 1. If $u=u(x, t)$ is a bounded solution of the equation 
(1.1) in $\Omega$ and $|u| \leqq K$, then for any two points $(x, t)$ and $(y, s) \in \Omega_{\delta}$, there exist positive constants $H$ and $\alpha$ such that

$$
|u(x, t)-u(y, s)| \leqq H\left(|x-y|+|t-s|^{1 / 2}\right)^{\alpha},
$$

where the constants $H$ and $\alpha$ depend on $n, a, \lambda, \mu, \varepsilon, K$ and $\delta$ alone. (See [7]).

Corollary 2. Let $\Omega_{\delta}$ (defined above) be a convex subdomain of the domain $\Omega$. If $u=u(x, t)$ is a positive solution of the equation (1. 1) in $\Omega$, then

$$
\log \frac{u(x, t)+L}{u(y, s)+L} \leqq r\left\{\frac{|x-y|^{2}}{s-t}+\frac{s-t}{\delta^{2}}+1\right\}
$$

for $(x, t),(y, s) \in \Omega_{\delta}, t<s$, where $\gamma$ is a positive constant depending only on $n, a, \varepsilon$ and $\lambda$, and

$$
L=\delta^{\eta}\|e\|_{\frac{n}{1-\varepsilon}}^{(R)}+\delta^{K}|f|_{\frac{n}{2-\varepsilon}}^{(R)}+\left(\delta^{K}\|g\|_{\frac{n}{2-\varepsilon}}^{(R)}\right)^{1 / 2} .
$$

(See [1]).

The essential contents of these results have been published before by the auther [14] [15] in Japanese but some of them are improved in this paper.

The proof of Theorem 1 shall be given according to the same argument by J. Moser [5] using estimates of solutions described in $\S 3$ and $\S 4$ but the proofs of Corollary 1 and 2 shall be given in the following paragraphs.

The Proof of Corollary 1. Let $R^{\prime}, R^{+}$and $R^{-}$be the domains defined in (2.2) with $k=1, k_{1}=1 / 2, h^{+}=3 / 4, h_{1}^{-}=1 / 3$ and $h_{2}^{-}=1 / 2$. We choose $\rho \leqq \delta$ arbitrarily. For a solution $v=v(x, t)$ which is nonnegative in $R^{\prime}$, we have

$$
\max _{R^{-}} v \leqq \gamma\left\{\min _{R^{+}} v+L(\rho)\right\}
$$

from (2.4) in Theorem 1. If we normalize the solution $v$ by the mean value;

$$
\mu^{-}=\frac{1}{\left|R^{-}\right|} \iint_{R^{-}} v(x, t) d x d t \leqq \max _{R^{-}} v
$$

then we obtain from (2.10) 


$$
\min _{R^{-}} v+L(\rho) \geqq r^{-1} \mu^{-} .
$$

Suppose that $u=u(x, t)$ is a bounded solution in $\Omega$ and we denote

$$
\begin{aligned}
& M=\max _{R^{\prime}} u, M^{+}=\max _{R^{+}} u \\
& m=\min _{R^{\prime}} u, m^{+}=\min _{R^{-}} u .
\end{aligned}
$$

Then

$$
v_{1}=M-u \text { and } v_{2}=u-m
$$

are solutions of the equations

$$
\operatorname{div} A_{i}\left(x, t, u, u_{x}\right)-u_{t}=B_{i}\left(x, t, u, u_{x}\right), i=1 \text { and } 2
$$

respectively, where

$$
\begin{aligned}
& A_{1}(x, t, u, p)=-A(x, t, M-u,-p) \\
& B_{1}(x, t, u, p)=-B(x, t, M-u,-p) \\
& A_{2}(x, t, u, p)=A(x, t, u+m, p) \\
& B_{2}(x, t, u, p)=B(x, t, u+m, p) .
\end{aligned}
$$

These functions defined above satisfy the assumptions (1.2) which are,

$$
\begin{aligned}
\left|A_{1}(x, t, u, p)\right| & \leqq a|-p|+c|M-u|+e \\
& \leqq a|p|+c|u|+(e+K c) \\
\left|B_{1}(x, t, u, p)\right| & \leqq b|p|+d|u|+(f+K d) \\
p \cdot A_{1}(x, t, u, p) & \geqq a^{-1}|-\not|^{2}-d|M-u|^{2}-g \\
& \geqq a^{-1}|p|^{2}-2 d|u|^{2}-\left(g+K^{2} 2 d\right),
\end{aligned}
$$

etc.

As the assumption (2.6) of the corollaries satisfy the condition (2.3) with respect to the equations (2.12), for solutions $v_{1}=M-u$ and $v_{2}=u-m$ being nonnegative in $R^{\prime}$, the inequality (2.11) implies that

$$
\begin{aligned}
& M-M^{+}+L(\rho) \geqq r^{-1}\left(M-\mu^{-}\right) \\
& m^{+}-m+L(\rho) \geqq r^{-1}\left(\mu^{-}-m\right)
\end{aligned}
$$

while

$$
\begin{aligned}
L(\rho) & =\rho^{\eta}\|e+K c\|_{\frac{n}{1-\varepsilon}}^{(R)}+\rho^{\kappa}\|f+K d\|_{\frac{n}{1-\varepsilon}}^{(R)}+\left(\rho^{\kappa}\left\|g+K^{2} 2 d\right\|_{\frac{n}{1-\varepsilon}}^{(R)}\right)^{1 / 2} \\
& \leqq \rho^{\varepsilon} 2^{-1} L_{0},
\end{aligned}
$$

where $L_{0}$ is a constant depending on $\delta, \lambda, \mu$ and $K$ alone. Therefore 
we have from (2.13)

$$
M^{+}-m^{+} \leqq\left(1-r^{-1}\right)(M-m)+L_{0} \rho^{\varepsilon} .
$$

Denoting the oscillation $M-m$ of $u$ in $R^{\prime}=R_{\rho, \rho^{2}}$ by $\omega(\rho)$, we may write (2.14) in the form;

$$
\omega\left(2^{-1} \rho\right) \leqq \theta \omega(\rho)+L_{0} \rho^{\varepsilon},
$$

where $\theta=1-\gamma^{-1}$ and $L_{0}$ are independent of $\rho$. Using the inequality above with $\rho=2^{-\nu} \rho(\nu=0.1,2, \cdots)$, we obtain a recursion

$$
\omega\left(2^{-\nu+1} \delta\right) \leqq \theta \omega\left(2^{-\nu} \delta\right)+L_{0}\left(2^{-\nu} \delta\right)^{\varepsilon}
$$

and hence the iteration of the recursion (2.15) yields

$$
\omega\left(2^{-\nu} \delta\right) \leqq \theta\left\{\omega(\delta)+\frac{L_{0} \delta^{\varepsilon}}{\theta} \sum_{i=1}^{\nu}\left(\frac{1}{2^{\varepsilon} \theta}\right)^{i-1}\right\}
$$

for any integer $\nu$. Now we choose $\gamma$ sufficiently large so that

$$
0<\left(2^{\varepsilon} \theta\right)^{-1}=2^{-\varepsilon}\left(1-\gamma^{-1}\right)^{-1}<1 .
$$

It follows from (2.16) that

$$
\omega\left(2^{-\nu} \delta\right) \leqq \theta^{\nu} \gamma_{0},
$$

where $\gamma_{0}$ is a constant depending on $n, a, \varepsilon, \lambda, \mu, \delta$ and $K$ alone.

We introduce for a point $x^{\prime}=(x, t)$ the following norm;

$$
\| x^{\prime}||= \begin{cases}\max \left\{\left|x_{1}\right|, \cdots,\left|x_{n}\right|, \sqrt{-t}\right\} & ; t<0 \\ \infty & ; t>0 .\end{cases}
$$

It suffices to prove (2.7) for $t<s$ in which case the inequality reads

$$
\left|u\left(x^{\prime}\right)-u\left(y^{\prime}\right)\right| \leqq H \mid\left\|x^{\prime}-y^{\prime}\right\|^{\alpha} \text {. }
$$

For $\left\|x^{\prime}-y^{\prime}\right\|>\delta,(2.18)$ is trivial since

$$
\left|u\left(x^{\prime}\right)-u\left(y^{\prime}\right)\right| \leqq 2 K \leqq 2 K \delta^{-\alpha} \|\left.\left|x^{\prime}-y^{\prime}\right|\right|^{\alpha} .
$$

For $\left\|\mid x^{\prime}-y^{\prime}\right\| \leqq \delta$, we choose an integer $\nu>0$ so that

$$
2^{-\nu-1} \delta \leqq \| x^{\prime}-y^{\prime}||<2^{-\nu} \delta
$$

and from (2.17), we have

$$
\left|u\left(x^{\prime}\right)-u\left(y^{\prime}\right)\right|<\theta^{\nu} \gamma_{0}=2^{-\alpha \nu} \gamma_{0} \leqq \frac{\| x^{\prime}-y^{\prime}||^{\alpha}}{\delta^{\alpha}} \gamma_{0}
$$

with $\alpha=-\log _{2} \theta$. This proves (2.7).

The Proof of Corollary 2. Applying Theorem 1 to the rectan- 
gle domain;

$$
\left|x_{i}\right|<\frac{1}{2} \rho, i=1, \cdots, n,-\frac{1}{4} \rho^{2}<t<\frac{3}{4} \rho^{2}
$$

with $\rho \leqq 2 \delta$. We find that for a positive solution $u$

$$
u(0,0) \leqq r\{u(x, t)+L(\rho)\},
$$

hence making use of the notation (2.9), we have

$$
u(0,0)+L \leqq r\{u(x, t)+L\}
$$

for a point $(x, t)$ such that

$$
\left|x_{i}\right|<\frac{1}{4} \rho, i=1, \cdots, n, t=\frac{1}{2} \cdot \rho^{2},
$$

where $\gamma$ is a constant depending on $n, a, \lambda, \varepsilon$ and $\delta$. While for two points $(0,0)$ and $(x, t)$ in $\Omega_{\sigma}$, we choose the points $\left(x^{\nu}, t^{\nu}\right)$ such that

$$
x_{\imath}^{\nu}=\nu x_{i} / N, i=1, \cdots, n, t^{\nu}=\nu t / N
$$

on the line, where

$$
\frac{\left|x_{i}\right|}{N}<\frac{1}{4} \rho, \frac{t}{N}=\frac{1}{2} \rho^{2} .
$$

since

$$
\frac{t}{\left|x_{i}\right|}=\frac{t / N}{\left|x_{i}\right| / N} \geqq \frac{\rho^{2} / 2}{\rho / 4}=2 \rho,
$$

we can choose an integer $\mathrm{N}$ and $\rho \leqq 2 \delta$ so that (2.20) holds. Therefore from (2.20) we have

$$
N=\frac{t}{\rho^{2} / 2}>\frac{2 t}{\delta^{2}}, N>\frac{8|x|^{2}}{t} .
$$

Applying (2.19) to the positive solution $u(x, t)$ in the rectangle domain;

$$
\left|x_{i}-x_{\imath}^{\nu}\right|<\frac{1}{2} \rho, i=1, \cdots, n,-\frac{1}{4} \rho^{2}<t-t^{\nu}<\frac{3}{4} \rho^{2},
$$

we obtain

$$
u\left(x^{\nu}, t^{\nu}\right)+L \leqq r\left\{\left(x^{\nu+1}, t^{\nu+1}\right)+L\right\}
$$

for $\nu=0,1, \cdots, N-1$ and therefore

$$
u(0,0)+L \leqq \gamma^{N}\{u(x, t)+L\}
$$


by multiplying the inequalities (2.22). Choosing $N$ as the smallest integer satisfying (2.21), we have

$$
\log \frac{u(0,0)+L}{u(x, t)+L} \leqq N \cdot \log \gamma \leqq \gamma\left\{\frac{|x|^{2}}{t}+\frac{t}{\delta^{2}}+1\right\}
$$

where $\gamma$ depends on $n, a, \lambda, \varepsilon$ and $\delta$ alone. This proves (2.8).

\section{§. Estimates of solutions (I)}

Inside the domain $R$ we denote by $R_{\rho \tau}$ a parallel rectangle subdomain;

$$
\left\{(x, t) ;(x, t) \in R,\left|x_{i}\right|<\rho, i=1, \cdots, n,-\tau<t<0\right\}=Q_{\rho} \times(-\tau, 0) .
$$

Here, we transformed the coordinates in $R$ such that the origin of the transformed coordinates should be on the center of the upper side of the rectangle subdomain $R_{\rho \tau}$.

To obtain results in this section and the next one, we need a useful inequality which has been known as Sobolev's Lemma;

Lemma 1. For a function $w=w(x)$ with compact support in $R^{n}$ whose derivatives $\partial w / \partial x_{i}(i=1, \cdots, n) \in L^{r}\left(R^{n}\right), r<n$, an inequality

$$
\|w\|_{L \frac{n r}{n-r}} \leqq \frac{r}{2} \cdot \frac{n-1}{n-r} \prod_{i=1}^{n}\left\|\partial w / \partial x_{i}\right\|_{L^{r}}^{\frac{1}{n}}
$$

holds.

Proof. See [8] [15j.

To formulate estimates of solutions, we define for a function $w=w(x, t)$,

$$
\begin{aligned}
& H_{\rho \tau}(w)=\rho^{-n} \tau^{-1} \iint_{R_{\rho \tau}} w(x, t)^{2} d x d t \\
& D_{\rho \tau}(w)=\rho^{-n+2} \tau^{-1} \iint_{R_{\rho \tau}} w_{x}(x, t)^{2} d x d t \\
& M_{\rho \tau}(w)=\rho^{-n} \max _{-\tau<t<0} \int_{Q_{\rho}}(x, t)^{2} d x,
\end{aligned}
$$

where $w_{x}^{2}=\left(\partial w / \partial x_{1}\right)^{2}+\cdots+\left(\partial w / \partial x_{n}\right)^{2}$.

As a consequence of Lemma 1, we have the following lemma;

Lemma 2. For a function $w=w(x, t)$ the values (3.2) of which exist, we have 


$$
H_{\rho \tau}\left(w^{\beta}\right) \leqq \gamma M_{\rho \tau}(w)^{\beta-1}\left\{H_{\rho \tau}(w)+D_{\rho \tau}(w)\right\}
$$

where

$$
\beta=\left\{\begin{array}{r}
1+2 / n ; n \geqq 3 \\
5 / 3 ; n=1,2
\end{array}\right.
$$

and $\gamma$ is a constant which depends only on $n$.

Proof. See [5].

Now, we shall introduce another parallel rectangle subdomain $R_{\rho \tau}^{*}$ of the domain $R$, that is,

$$
R_{\rho \tau}^{*}=\left\{(x, t) ;(x, t) \in R,\left|x_{i}\right|<\rho, i=1, \cdots, n, 0<t<\tau\right\}=Q_{\rho} \times(0, \tau) .
$$

Lemma 3. (1) If $u=u(x, t)$ is a nonnegative subsolution of the equation (1.1) in $R_{\rho \tau}$, then

$$
\begin{aligned}
& D_{\rho^{\prime} \tau^{\prime}}(v) \leqq \gamma p^{4 /(1+\varepsilon)}\left(\frac{p}{p-1}\right)^{2} \Phi H_{\rho \tau}(v) \\
& M_{\rho^{\prime} \tau^{\prime}}(v) \leqq \gamma p^{4 /(1+\varepsilon)}\left(\frac{p}{p-1}\right) \Psi H_{\rho \tau}(v)
\end{aligned}
$$

for $v=\{u+L(\rho)\}^{p / 2}, p>1$ and $R_{\rho^{\prime} z^{\prime}} \subset R_{\rho \tau}$.

(2) If $u=u(x, t)$ is a nonnegative supersolution of the equation (1.1) in $R_{\rho \tau}^{*}$, then

$$
\begin{aligned}
& D_{\rho^{\prime} \tau^{\prime}}(v) \leqq r\left\{\left(\frac{p}{1-p}\right)^{2}+\left(\frac{p}{1-p}\right)\right\}^{(1-\varepsilon) / 2} \Phi H_{\rho \tau}(v) \\
& M_{\rho^{\prime} \tau^{\prime}}(v) \leqq r\left\{\left(\frac{p}{1-p}\right)+\left(\frac{1-p}{p}\right)\right\}^{(1+\varepsilon) / 2} \Psi H_{\rho \tau}(v)
\end{aligned}
$$

for $v(x, t)=\{u(x,-t)+L(\rho)\}^{p / 2} ; 0<p<1$ and $R_{\rho^{\prime} \tau^{\prime}} \subset R_{\rho^{\tau} \text {. }}$

(3) If $u=u(x, t)$ is a positive supersolution of the equation (1.1) in $R_{\rho \tau}$, then

$$
\begin{aligned}
& D_{\rho^{\prime} \tau^{\prime}}(v) \leqq \gamma\left(1+|p|^{2 / \varepsilon}\right) \Phi H_{\rho \tau}(v) \\
& M_{\rho^{\prime} \tau^{\prime}}(v) \leqq \gamma\left(1+|p|^{2 / \varepsilon}\right) \Psi H_{\rho \tau}(v)
\end{aligned}
$$

for $v=\{u+L(\rho)\}^{p / 2}, p<0$ and $R_{\rho^{\prime} \tau^{\prime}} \subset R_{\rho 7}$. Here, $r$ is a positive constant which depends on $n, a, \lambda$ and $\varepsilon$ alone and

$$
\begin{aligned}
& \Phi=\left\{\frac{\rho^{\prime 2}}{\tau-\tau^{\prime}}+\frac{\rho^{\prime 2}}{\left(\rho-\rho^{\prime}\right)^{2}}\right\}\left(\frac{\rho}{\rho^{\prime}}\right)^{n}\left(\frac{\tau}{\tau^{\prime}}\right) \\
& \Psi=\left\{\frac{\tau^{\prime}}{\tau-\tau^{\prime}}+\frac{\tau^{\prime}}{\left(\rho-\rho^{\prime}\right)^{2}}\right\}\left(\frac{\rho}{\rho^{\prime}}\right)^{n}\left(\frac{\tau}{\tau^{\prime}}\right) .
\end{aligned}
$$


The proof of Lemma 3 will be given later.

We denote $\bar{u}=|u|+L(\rho)$ for a solution (or subsolution or supersolution) $u=u(x, t)$ of the equation (1.1) in $R$ and we introduce the following norm;

$$
M(p, D)=\left(\frac{1}{|D|} \iint_{D} \bar{u}^{p} d x d t\right)^{1 / p}
$$

for a rectangle subdomain $D$ of $R^{\prime}$ and $-\infty<p<+\infty$, where $|D|$ is the measure of $D$. It can be easily known that $M(p, D)$ is increasing as a function of $p>0$ and interpolates $M(\infty, D)=\max _{D} u$ and $M(-\infty$, $D)=\min _{D} u$. See $[15]$. We aim at obtaining in this section the main theorem as follows;

Theorem 2. (1) If $u=u(x, t)$ is a subsolution of the equation

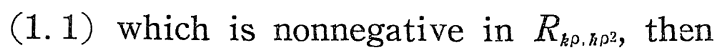

$$
M\left(\infty, R_{k^{\prime} \rho, h^{\prime} \rho^{2}}\right) \leqq \gamma M\left(p, R_{k \rho, h \rho^{2}}\right)
$$

for $p \geqq p_{0}>1$, where $\gamma$ is a positive constant which depends on $n, a, \lambda$, $\varepsilon, k, k^{\prime}, h, h^{\prime}$ and $p_{0}$ alone.

(2) If $u=u(x, t)$ is a supersolution of the equation (1.1) which is nonnegative in $R_{k_{\rho}, h_{\rho}}^{*}$, then

$$
M\left(p_{0}, R_{k^{\prime} \rho, h^{\prime} \rho^{2}}^{*}\right) \leqq \gamma M\left(\delta, R_{k \rho, h \rho^{2}}\right)
$$

for a $\delta>0$, where

$$
p_{0}=\left\{\begin{array}{cl}
1+(1 / n) & ; n \geqq 3 \\
4 / 3 & ; n=1 \text { or } 2
\end{array}\right.
$$

and $\gamma$ is a positive constant which depends on $n, a, \lambda, \varepsilon, k, k^{\prime}, h, h^{\prime}$ and $\delta$ alone.

(3) If $u=u(x, t)$ is a supersolution of the equation (1.1) which is positive in $R_{k \rho, h \rho^{2}}$, then

$$
M\left(-\delta, R_{k \rho, h \rho^{2}}\right) \leqq \gamma M\left(-\infty, R_{k^{\prime} \rho, h^{\prime} \rho 2}\right)
$$

for any $\delta>0$, where $\gamma$ is a positive constant which depends on $n, a, \lambda$, $\varepsilon, k, k^{\prime}, h, h^{\prime}$ and $\delta$ alone.

The above inequalities (3.10), (3.11) and (3.12) in Theorem 2 can be derived using the estimates (3.5), (3.6) and (3.7) in Lemma 
3 respectively. (c.f. [5], [15]) Now, we shall begin with proving Lemma 3.

The Proof of Lemma 3. (1) From the assumption (1.2), we obtain for $\bar{u}=|u|+L(\rho)$

$$
\begin{aligned}
& |A| \leqq a_{\mid} p \mid+\bar{c} \bar{u} \\
& |B| \leqq b|p|+\bar{d} \bar{u} \\
& p \cdot A \geqq a^{-1}|p|^{2}-\bar{d} \bar{u}^{2}
\end{aligned}
$$

where we denote $L(\rho)$ by (2.5) and

$$
\begin{aligned}
& \bar{c}=c+L(\rho)^{-1} e \\
& \bar{d}=d+L(\rho)^{-1} f+L(\rho)^{-2} g .
\end{aligned}
$$

While from the assumption (2.3) we have

$$
\begin{aligned}
& \rho^{\eta}\|\bar{c}\| \leqq \rho^{\eta}\|c\|+L(\rho)^{-1} \rho^{\eta}\|e\| \leqq \lambda+1 \\
& \rho^{\kappa}\|\bar{d}\| \leqq \rho^{\kappa}\|d\|+L\left({ }^{\prime}\right)^{-1} \rho^{\kappa}\|f\|+L(\rho)^{-2} \rho^{\kappa}\|g\| \leqq \lambda+2 .
\end{aligned}
$$

Now, for a nonnegative subsolution $u$ in $R_{\rho r}$, we choose as a test function of the integral inequality (2.1)

$$
\phi=p \bar{u}^{p-1} \psi^{2}, p>1, \bar{u}=|u|+L(\rho),
$$

where $\psi=\psi(x, t)$ is any function piecewise differentiable and vanishing both on the boundary and in the exterior of $R_{\rho \tau}$ except possibly at $t=0$. Then, from (3.13)

$$
\begin{aligned}
& \phi u_{t}+\phi_{x} \cdot A+\phi B \\
& \geqq p \bar{u}^{p-1} \psi^{2} \bar{u}_{t}+a^{-1} p(p-1) \bar{u}^{p-2}\left|\bar{u}_{x}\right|^{2} \psi^{2} \\
& -\bar{d} \bar{u}^{2} p(p-1) \bar{u}^{p-2} \psi^{2}-a\left|u_{x}\right| 2 p \bar{u}^{p-1} \psi\left|\psi_{x}\right| \\
& -\bar{c} \bar{u} \cdot 2 p \bar{u}^{p-1} \psi\left|\psi_{x}\right|-b\left|\bar{u}_{x}\right| p \bar{u}^{p-1} \psi^{2} \\
& -\bar{d} \bar{u} \cdot p \bar{u}^{p-1} \psi^{2} .
\end{aligned}
$$

The right hand side of the inequality above can be expressed in terms of $v=\bar{u}^{\frac{p}{2}}$ as follows;

$$
\begin{aligned}
& =\left(\psi^{2} v^{2}\right)_{t}-2 \psi \psi_{t} v^{2}+4 \frac{p-1}{p a} \psi^{2} v_{x}^{2}-\bar{d} p(p-1) \psi^{2} v^{2} \\
& -4 a \psi\left|\psi_{x}\right| v\left|v_{x}\right|-2 p \bar{c} \psi\left|\psi_{x}\right| v^{2}-2 b \psi^{2} v\left|v_{x}\right|-\overline{d p} \psi^{2} v^{2}
\end{aligned}
$$

Therefore the definition of a subsolution (2.1) implies that

$$
\iint_{R_{\rho \tau}}\left\{\left(\psi^{2} v^{2}\right)_{t}+4 \frac{p-1}{p a} \psi^{2} v_{x}^{2}\right\} d x d t
$$




$$
\begin{aligned}
\leqq & \iint_{R_{\rho r}} \\
& \left\{2 \psi_{r}\left|\psi_{t}\right| v^{2}+4 a \psi r\left|\psi_{x}\right| v\left|v_{x}\right|+2 b \psi_{r}^{2} v_{\mid}\left|v_{x}\right|\right. \\
& \left.+2 p \cdot \bar{c} \psi_{r}\left|\psi_{x}\right| v^{2}+p^{2} \bar{d} \psi^{2} v^{2}\right\} d x d t
\end{aligned}
$$

For the second term of the right hand side of the inequality (3.17), we have

$$
\text { (3. 18) } \iint 4 a \psi\left|\psi_{x}\right| v\left|v_{x}\right| d x d t \leqq \frac{p-1}{p a} \iint \psi^{2} v_{x}^{2} d x d t+\frac{4 p a^{3}}{p-1} \iint \psi_{x}^{2} v^{2} d x d t \text {. }
$$

To estimate the third term of the same inequality (3.17), we assume that $n \geqq 3$. By using Sobolev's inequality (3.1) with $r=2$ in Lemma 1 , and the condition (2.3), we have

$$
\begin{aligned}
& \int_{Q_{\rho}} b \psi^{2} v\left|v_{x}\right| d x=\int b(\psi v)^{\varepsilon}(\psi v)^{1-\varepsilon}\left|\psi r v_{x}\right| d x \\
& \leqq \rho^{\varepsilon}\left(\int_{Q_{\rho}} b^{\frac{n}{1-\varepsilon}} d x\right)^{\frac{1-\varepsilon}{n}}\left(\frac{1}{\rho^{2}} \int_{Q_{\rho}}(\psi v)^{2} d x\right)^{\frac{\varepsilon}{\rho^{2}}}\left(\int_{R^{n}}(\psi v)^{\frac{2 n}{n-2}} d x\right)^{\frac{(n-2)(1-\varepsilon)}{2 n}} \\
& \qquad\left(\int_{Q_{\rho}}\left|\psi v_{x}\right|^{2} d x\right)^{1 / 2} \\
& \leqq \lambda \gamma\left(\frac{1}{\rho^{2}} \int_{Q_{\rho}} \psi^{2} v^{2} d x\right)^{\varepsilon / 2}\left(\int_{R^{n}}(\psi v)_{x}^{2} d x\right)^{\frac{1-\varepsilon}{2}}\left(\int_{Q_{p}} \psi^{2} v_{x}^{2} d x\right)^{1 / 2} \\
& \leqq \gamma_{1} \mu^{\frac{-2+\varepsilon}{2}}\left\{\frac{\varepsilon}{2} \cdot \frac{1}{\rho^{2}} \int_{Q_{\rho}} \psi^{2} v^{2} d x+\frac{1-\varepsilon}{2} \mu \int \psi^{2} v_{x}^{2} d x\right. \\
& \left.\quad+\frac{1-\varepsilon}{2} \cdot \mu \int \psi_{x}^{2} v^{2} d x+\frac{1}{2} \mu \int \psi^{2} v_{x}^{2} d x\right\}
\end{aligned}
$$

Here, $\gamma_{1}$ is a constant depending only on $n$ and $\lambda . \mu$ is an arbitrary constant, which should be decided such that

$$
\gamma_{1} \cdot \frac{2-\varepsilon}{2} \mu^{\frac{-2+\varepsilon}{2}+1}=-\frac{p-1}{2 p a}
$$

that is,

$$
\mu=\gamma_{2} \cdot\left(\frac{p-1}{p}\right)^{2 / \varepsilon}
$$

where $\gamma_{2}$ is also a constant depending on $n, \lambda$ and $\varepsilon$ alone. Then, we establish

$$
\iint_{R_{\rho \tau}} 2 b \psi r^{2} v\left|v_{x}\right| d x d t \leqq \frac{p-1}{2 p a} \iint_{R_{\rho \tau}} \psi^{2} v_{x}^{2} d x d t
$$




$$
+r\left(\frac{p}{p-1}\right) \iint_{R_{\rho \tau}}\left\{\psi_{x}^{2}+\rho^{-2} \psi^{2}\right\} v^{2} d x d t
$$

The case of $n=1$ or 2 is contained in that for $n=3$ by ignoring two or one variables. By the same arguments for the forth and the fifth term of the right hand side of the inequality (3.17), we have respectively

$$
\begin{aligned}
\iint_{R_{\rho \tau}} 2 p \bar{c} \psi\left|\psi_{x}\right| v^{2} d x d t \leqq \frac{p-1}{2 p a} \iint_{R_{\rho \tau}} \psi^{2} v_{2}^{x} d x d t \\
+r \cdot p^{\frac{2}{1+\varepsilon}}\left(\frac{p}{p-1}\right) \iint_{R_{\tau \rho}}\left\{\psi_{x}^{2}+\rho^{-2} \psi^{2}\right\} v^{2} d x d t \\
\iint p^{2} \bar{d} \psi^{2} v^{2} d x d t \leqq \frac{p-1}{p a} \iint_{R_{\rho \tau}} \psi^{2} v_{x}^{2} d x d t \\
+r \cdot p^{\frac{4}{1+\varepsilon}}\left(\frac{p}{p-1}\right) \iint_{R_{\rho \tau}}\left\{\psi_{x}^{2}+\rho^{-2} \psi^{2}\right\} v^{2} d x d t .
\end{aligned}
$$

Therefore by making use of estimates (3.18), (3.19), (3.20) and (3.21), we establish from (3.17)

$$
\begin{aligned}
& \iint_{R_{\rho \tau}}\left\{\left(\psi^{2} v^{2}\right)_{t}+\frac{p-1}{p a} \psi^{2} v_{x}^{2}\right\} d x d t \\
& \leqq r \cdot p^{\frac{4}{1+\varepsilon}}\left(\frac{p}{p-1}\right) \iint_{R_{\rho \tau}}\left\{\psi\left|\psi_{t}\right|+\psi_{x}^{2}+\rho^{-2} \psi^{2}\right\} d x d t .
\end{aligned}
$$

Now, we choose as $\psi$ in (3.22) or (3.16) a function $\psi(x, t)=\psi_{0}(t)$. $\prod_{i=1}^{n} \psi_{i}\left(\left|x_{i}\right|\right)$, where

$$
\begin{aligned}
& \psi_{0}(t)=\left\{\begin{array}{cl}
1 & ;-\tau^{\prime} \leqq t \leqq 0 \\
\frac{\tau+t}{\tau+\tau^{\prime}} & ;-\tau<t<-\tau^{\prime} \\
0 & ; t \leqq-\tau \text { or } 0<t
\end{array}\right. \\
& \psi_{i}(r)=\left\{\begin{array}{cl}
1 & ; 0 \leqq r \leqq \rho^{\prime} \\
\frac{\rho-r}{\rho-\rho^{\prime}} & ; \rho^{\prime}<r<\rho \\
0 & ; \rho \leqq r .
\end{array}\right.
\end{aligned}
$$

The inequality (3.22) implies that

$$
\iint_{R_{\rho^{\prime} \tau^{\prime}}} v_{x}^{2} d x d t \leqq \gamma \cdot p^{\frac{4}{1+\varepsilon}}\left(\frac{p}{p-1}\right)^{2}\left\{\frac{1}{\tau-\tau^{\prime}}+\frac{1}{\left(\rho-\rho^{\prime}\right)^{2}}\right\} \iint_{R_{\rho \tau}} v^{2} d x d t
$$

Since $\psi$ was not required to vanish on the upper boundary, we consider 
the rectangle domain

$$
\begin{array}{r}
R_{\rho \tau}^{\sigma}=\left\{(x, t) ;(x, t) \in R_{\rho \tau}, \quad\left|x_{i}\right|<\rho i=1, \cdots, n,\right. \\
\left.-\tau<t<-\sigma,-\tau<-\tau^{\prime}<-\sigma<0\right\}
\end{array}
$$

and choose $\sigma$ such that

$$
\left.\int_{Q_{\rho^{\prime}}} v^{2} d x\right|_{\tau=-\sigma} \geqq \frac{1}{2} \max _{-\tau^{\prime}<t<0} \int_{Q_{\rho^{\prime}}} v^{2} d x
$$

Then, we have

$$
\begin{aligned}
& \max _{-\tau^{\prime}<t<0} \int_{Q_{\rho^{\prime}}} v^{2} d x \leqq\left. 2 \int_{Q_{\rho^{\prime}}} v^{2} d x\right|_{t=-\sigma} \\
& \leqq 2 \int_{Q_{\rho}} \psi^{2} v^{2} d x \mid \begin{array}{l}
t=-\sigma=2 \iint_{R_{\rho \tau}^{\sigma}}\left(\psi^{2} v^{2}\right)_{t} d x d t \\
t=-\tau
\end{array} \\
& \leqq r \cdot p^{\frac{4}{1+\varepsilon}}\left(\frac{p}{p-1}\right)\left\{\frac{1}{\tau-\tau^{\prime}}+\frac{1}{\left(\rho-\rho^{\prime}\right)^{2}}\right\} \iint v^{2} d x d t .
\end{aligned}
$$

Inequalities (3.23) and (3.24) are nothing but (3.5) for us to derive.

(2), (3). We can prove (2) and (3) of Lemma 3 by the same arguments as that of (1) but for choosing as a test function of the inequality (2.1)

$$
\phi=\phi(x,-t)=p \bar{u}(x,-t)^{p-1} \psi(x, t)^{2}, \quad 0<p<1
$$

and

$$
\phi=\phi(x, t)=-p \bar{u}^{p-1} \psi(x, t)^{2}, \quad p<0
$$

in the case of (2) and (3) respectively instead of the test function (3.16) in (1).

The Proof of Theorem 2. (1) Setting $v=\bar{u}^{p / 2}, p \geqq p_{0}>1$, for a subsolution $u$ of the equation (1.1), we apply Lemma 3 (1) with respect to $R_{k^{\prime} \rho, h^{\prime} \rho^{2}} \subset R_{k \rho, h \rho 2}$. Hence we have

$$
\begin{aligned}
D_{k^{\prime} \rho, h^{\prime} \rho 2}(v) \leqq & r p^{\frac{4}{1+\varepsilon}}\left(\frac{p}{p-1}\right)^{2}\left\{\frac{k^{\prime 2}}{h-h^{\prime}}+\frac{k^{\prime 2}}{\left(k-k^{\prime}\right)^{2}}\right\} \\
& \times\left(\frac{k}{k^{\prime}}\right)^{n}\left(\frac{h}{h^{\prime}}\right) H_{k \rho, h \rho^{2}}(v) \\
M_{k^{\prime} \rho, h^{\prime} \rho^{2}}(v) \leqq & r p^{\frac{4}{1+\varepsilon}}\left(\frac{p}{p-1}\right)\left\{\frac{h^{\prime}}{h-h^{\prime}}+\frac{h^{\prime}}{\left(k-k^{\prime}\right)^{2}}\right\} \\
& \times\left(\frac{k}{k^{\prime}}\right)^{n}\left(\frac{h}{h^{\prime}}\right) H_{k \rho, h \rho 2}(v),
\end{aligned}
$$


where $\gamma$ is a constant depending only on $n, a, \lambda$ and $\varepsilon$. Applying (3.25) to the formula (3.3) in Lemma 2 , we obtain

$$
H_{k^{\prime}, k^{\prime} \rho^{\prime} 2}\left(v^{\beta}\right) \leqq \gamma_{1} \cdot H_{k \rho, k \rho \rho^{2}}(v)^{\beta},
$$

where

$$
\begin{gathered}
\gamma_{1}=r p^{\frac{4}{1+\varepsilon} \cdot \beta}\left(\frac{p}{p-1}\right)^{2 \beta}\left\{\frac{1}{h-h^{\prime}}+\frac{1}{\left(k-k^{\prime}\right)^{2}}+1\right\} \\
\quad \times\left(1+k^{\prime 2}\right) h^{\prime \beta-1}\left(\frac{k}{k^{\prime}}\right)^{n \beta}\left(\frac{h}{h^{\prime}}\right)^{\beta}
\end{gathered}
$$

and $\gamma$ is a constant depending only on $n, a, \lambda$ and $\varepsilon$. Now, we define

$$
\begin{aligned}
& p_{\nu}=p_{0} \beta^{\nu}, \quad v_{\nu}=\bar{u}^{\frac{p_{\nu}}{2}} \\
& k_{\nu}=k \cdot \frac{1+k^{\prime} \nu}{1+k_{\nu}}, \quad h_{\nu}=h \cdot \frac{1+h^{\prime} \nu}{1+h_{\nu}} \\
& H_{\nu}=H_{k_{\nu}, k_{\nu} \rho^{2}}\left(v_{\nu}\right)
\end{aligned}
$$

for $\nu=0,1,2, \cdots$. Then the inequality (3.26) implies a recursion formula

$$
H_{\nu+1} \leqq \gamma_{(\nu)} H_{\nu}^{\beta},
$$

whence the constant $\gamma_{(\nu)}$ can be estimated in the form;

$$
\gamma(\nu) \leqq \gamma^{\nu+1}
$$

for some constant $\gamma$ depending on $n, a, \lambda, \varepsilon, k, k^{\prime}, h, h^{\prime}$ and $p_{0}$ alone from the expression (3.27) of the constant $\gamma_{1}$. Iteration of the recursion (3.28) and (3.29) gives

$$
H_{\nu} \leqq \gamma^{\nu+\beta(\nu-1)+\beta^{2}(\nu-2)+\cdots+\beta^{\nu-1}} H_{0}^{\beta^{\nu}} \leqq \gamma^{\beta^{\nu}} \cdot H_{0}^{\beta^{\nu}}
$$

which implies that

$$
\begin{aligned}
& \left(\frac{1}{\left|R_{k^{\prime} \rho, h^{\prime} \rho 2}\right|} \iint_{R_{k^{\prime} \rho, h^{\prime} \rho 2}}{\overline{u^{\prime}}}^{p_{0 \beta^{u}}} d x d t\right)^{\frac{1}{p_{0} \beta^{v}}}
\end{aligned}
$$

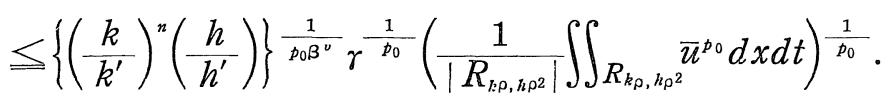

Rewriting the inequality above by making use of the definition (3.9), we have

$$
M\left(p_{0} \beta^{\nu}, R_{k^{\prime} \rho, h^{\prime} \rho 2}\right) \leqq \gamma^{\beta^{-\nu}} \cdot \gamma^{p_{0}^{-1}} M\left(p_{0}, R_{k \rho, k \rho p}\right) .
$$

When we let $\nu$ tend to $+\infty$ in (3.30) and use the properties of the 
norm $M(\not, D)$ in the definition (3.9), we establish the inequality (3.10) which is our result of Theorem 2, (1).

We shall be allowed not to prove Theorem 2, (2) and (3) since we can derive the results (3.11) and (3.12) from the inequalities (3.6) and (3.7) in Lemma 3 respectively by the arguments parallel to that of (1). (See [5], [14], [15]).

\section{§4. Estimates of solutions (II)}

We denote by $R_{k \rho,\left(h_{1}+h_{2}\right) \rho^{2}}$ a parallel rectangle subdomain

$$
\left\{(x, t) ;\left|x_{i}\right|<k_{\rho}, i=1, \cdots, n,-h_{2} \rho^{2}<t<h_{1} \rho^{2} .\right\}
$$

in the domain $R$. Furthermore we define a parallel rectangle subdomain $U$ and its subdomain $U^{+}$and $U^{-}$in the domain $R_{k \rho_{,}\left(k_{1}+k_{2}\right) \rho^{2}}$, as follows;

$$
\begin{aligned}
& U=\left\{(x, t) ;\left|x_{i}\right|<k^{\prime} \rho, i=1, \cdots, n,-h_{2}^{\prime} \rho^{2}<t<h_{1}^{\prime} \rho^{2}\right\} \\
& U^{+}=\left\{(x, t) ;\left|x_{i}\right|<k^{\prime} \rho, i=1, \cdots, n, 0<t<h_{1}^{\prime} \rho^{2}\right\} \\
& U^{-}=\left\{(x, t) ;\left|x_{i}\right|<k^{\prime} \rho, i=1, \cdots, n,-h_{2}^{\prime} \rho^{2}<t<0\right\}
\end{aligned}
$$

where $0<k^{\prime}<k, 0<h_{1}^{\prime} \leqq h_{1}, 0<h_{2}^{\prime}<h_{2}$.

For a real number $\sigma$, the following function $\Phi(\sigma)$ will be used in deriving the last estimate in Lemma 4.

$$
\Phi(\sigma)=\left\{\begin{array}{cl}
\sqrt{\bar{\sigma}} & ; \sigma \geqq 0 \\
0 & ; \sigma<0
\end{array}\right.
$$

Lemma 4. If $u=u(x, t)$ is a positive supersolution of the equation (1.1) in $R_{k \rho,\left(h_{1}+h_{2}\right) \rho^{2}}$, then

$$
\frac{1}{\left|U^{+}\right| \cdot\left|U^{-}\right|} \iint_{x^{\prime}=U^{+}, y^{\prime} \in U^{-}} \Phi\left\{v\left(x^{\prime}\right)-v\left(y^{\prime}\right)\right\} d x^{\prime} d y^{\prime} \leqq \gamma
$$

for $v=-\log \{u+L(\rho)\}$ and $U^{+}, U^{-} \subset U \subset R_{k \rho_{1}\left(h_{1}+h_{2}\right) \rho^{2}}$, where $x^{\prime}=(x, t)$, $d x^{\prime}=d x d t$ ahd $\gamma$ is a positive constant depending on $n, a, \lambda, \varepsilon, k^{\prime}, k, h_{1}^{\prime}$ and $h_{2}^{\prime}$ alone.

Theorem 3. If $u=u(x, t)$ is a supersolution of the equation (1.1) which is positive in $R_{k \rho,\left(h_{1}+h_{2}\right) \rho^{2} \text {, then }}$

$$
M\left(\alpha, C^{-}\right) \leqq r M\left(-\alpha, C^{+}\right)
$$


for subdomains $C^{-}$and $C^{+}$of $R_{k^{\prime} \rho\left(l_{1}^{\prime}+h^{\prime}\right) \rho^{2}}$ such that

$$
\begin{aligned}
& C^{+}=\left\{(x, t) ;\left|x_{i}\right|<k^{\prime} \rho, i=1, \cdots, n, h_{3}^{\prime} \rho^{2}<t<h_{1}^{\prime} \rho^{2} .\right\} \\
& C^{-}=\left\{(x, t) ;\left|x_{i}\right|<k^{\prime} \rho, i=1, \cdots, n,-h_{2}^{\prime} \rho^{2}<t<-h_{3}^{\prime} \rho^{2} .\right\}
\end{aligned}
$$

where $0<h_{3}^{\prime}<h_{1}^{\prime}<h_{1}, 0<h_{3}^{\prime}<h_{2}^{\prime}<h_{2}, 0<k^{\prime}<k$ and $\gamma$ and $\alpha$ are positive constants depending on $n, a, \lambda, \varepsilon, k^{\prime}, k, h_{1}^{\prime}, h_{2}^{\prime}, h_{3}^{\prime}, h_{1}$ and $h_{2}$ alone.

To prove Lemma 4 we need the following lemma;

Lemma 5. Let $p(x) \geqq 0$ be continuous of compact support of breadth $B$ and such that the domains $p(x) \geqq$ const. are convex. Then for any function $f(x)$ for which $f$ and $f_{x}$ are square integrable with respect to $p(x) d x$ one has

$$
\int\{f(x)-k\}^{2} p(x) d x \leqq c B^{2} \int f_{x}^{2} p(x) d x
$$

where

$$
c=\frac{\operatorname{Max} p(x)}{2 \int p(x) d x} \int_{p>0} d x, k=\frac{\int f(x) p(x) d x}{\int p d x} .
$$

The proof of Lemma 5 can be seen in [5].

The Proof of Lemma 4. It suffices to give the proof for $u \geqq \delta>0$ and prove the inequality (4.2) with $\gamma$ independent of $\delta$. For a supersolution $u$, we set

$$
v=-\log \bar{u}, \bar{u}=|u|+L(\rho)
$$

and choose as a test function $\phi$ in the inequality (2.1) the following one

$$
\phi=\phi(x, t)=\bar{u}(x, t)^{-1} \psi(x)^{2}
$$

where $\psi(x)$ is a function of compact support in $x$. Then using (3.13) which was derived from the assumption (1.2), we have

$$
\begin{aligned}
& \phi u_{t}+\phi_{x} \cdot A+\phi B \\
& \quad \leq \psi^{2} \bar{u}^{-1} \bar{u}_{t}-a^{-1} \psi^{2} \bar{u}^{-2}\left|\bar{u}_{x}\right|^{2}+\bar{d}_{\psi^{2}} \bar{u}^{-2} \bar{u}^{2} \\
& \quad+2 a \psi\left|\psi_{x}\right| \bar{u}^{-1}\left|\bar{u}_{x}\right|+2 \bar{c} \psi\left|\psi r_{x}\right| \bar{u}^{-1} \bar{u} \\
& \quad+b \psi^{2} \bar{u}^{-1}\left|\bar{u}_{x}\right|+\bar{d} \psi^{2} \bar{u}^{-1} \bar{u} \\
& \quad=-\psi^{2} v_{t}-a^{-1} \psi^{2} v_{x}^{2}+2 a \psi\left|\psi r_{x}\right|\left|v_{x}\right|+b \psi^{2}\left|v_{x}\right|
\end{aligned}
$$




$$
+2 \bar{c} \psi\left|\psi \psi_{x}\right| 2 \bar{d} \psi^{2}
$$

Since the given $u$ is a supersolution of the equation (1.1), we obtain from (4.6)

$$
\begin{aligned}
& \left.\int_{Q_{k P}} \psi^{2} v d x\right|_{t_{2}} ^{t_{1}}+a^{-1} \iint_{Q_{k \rho} \times\left(t_{1}, t_{2}\right)} \psi^{2} v_{x}^{2} d x d t \\
& \leqq \iint_{Q_{k_{\rho} \times\left(t_{1}, t_{2}\right)}}\left\{2 a \psi\left|\psi \psi_{x}\right|\left|v_{x}\right|+b \psi^{2}\left|v_{x}\right|+2 \bar{c} \psi\left|\psi_{x}\right|+2 \bar{d} \psi^{2}\right\} d x d t
\end{aligned}
$$

by integrating over $Q_{k p} \times\left(t_{1}, t_{2}\right)$ in (2.1). Now we shall estimate each term of the right hand side of the inequality (4.7) by the same arguments as that of the proof of Lemma 3 using Schwarz's or Hölder's inequality and Sobolev's inequality in Lemma 1.

For the first term of the right hand side of (4.7), we have

$$
\begin{aligned}
& \iint 2 a \psi\left|\psi_{x}\right|\left|v_{x}\right| d x d t \\
& \leqq \frac{1}{4 a} \iint \psi^{2} v_{x}^{2} d x d t+4 a^{3} \iint \psi_{x}^{2} d x d t .
\end{aligned}
$$

For the second term, we have from the assumption (2.3)

$$
\begin{aligned}
& \quad \int_{\alpha_{\beta}} b \psi^{2}\left|v_{x}\right| d x=\int b \psi^{\varepsilon} \psi^{1-\varepsilon}\left|\psi v_{x}\right| d x \\
& \leqq\left(k_{\rho}\right)^{\varepsilon}\left(\int b^{\frac{n}{1-\varepsilon}} d x\right)^{\frac{1-\varepsilon}{n}}\left(\frac{1}{k^{2} \rho^{2}} \int \psi^{2} d x\right)^{\varepsilon / 2}\left(\int \psi^{\frac{2 n}{n-2}} d x\right)^{\frac{(n-2)(1-\varepsilon)}{2 n}} \\
& \quad \times\left(\int \psi^{2} v_{x}^{2} d x\right)^{1 / 2} \\
& \leqq \lambda \cdot \mu^{-1 / 2}\left(\frac{1}{k^{2} \rho^{2}} \int \psi \psi^{2} d x\right)^{\varepsilon / 2}\left(\int \psi r_{x}^{2} d x\right)^{\frac{1-\varepsilon}{2}}\left(\mu \int \psi^{2} v_{x}^{2} d x\right)^{1 / 2} \\
& \leqq \lambda\left\{\mu^{-1 / 2} \frac{\varepsilon}{2} \cdot \frac{1}{k^{2} \rho^{2}} \int \psi^{2} d x+\mu^{-1 / 2} \cdot \frac{1-\varepsilon}{2} \int \psi_{x}^{2} d x+\mu^{1 / 2} \cdot \frac{1}{2} \int \psi^{2} v_{x}^{2} d x\right\} .
\end{aligned}
$$

Then, choosing a constant $\mu$ such as

$$
\frac{\lambda}{2} \mu^{1 / 2}=\frac{1}{4 a}, \text { that is, } \mu=\left(\frac{1}{2 a \lambda}\right)^{2} .
$$

we obtain

$$
\begin{aligned}
\iint b \psi^{2}\left|v_{x}\right| d x d t & \leqq \frac{1}{4 a} \iint \psi^{2} v_{x}^{2} d x d t \\
& +r \iint\left\{k^{-2} \rho^{-2} \psi^{2}+\psi_{x}^{2}\right\} d x d t
\end{aligned}
$$


In the same way we obtain estimates for the third and the forth term respectively as follows;

$$
\begin{aligned}
& \iint 2 \bar{c} \psi\left|\psi_{x}\right| d x d t \leqq r \iint\left\{k^{-2} \rho^{-2} \psi^{2}+\psi_{x}^{2}\right\} d x d t \\
& \iint \overline{d \psi^{2}} d x d t \leqq r \iint\left\{k^{-2} \rho^{-2} \psi^{2}+\psi_{x}^{2}\right\} d x d t .
\end{aligned}
$$

Therefore

$$
\begin{gathered}
\left.\iint_{Q k \rho} \psi^{2} v d x\right|_{t_{1}} ^{t_{2}}+\iint_{Q_{k_{\rho} \times\left(t_{1}, t_{2}\right)}} \psi^{2} v_{x}^{2} d x d t \\
\leqq \gamma \iint\left\{k^{-2} \rho^{-2} \psi^{2}+\psi_{x}^{2}\right\} d x d t
\end{gathered}
$$

is derived by applying the estimates (4.8), (4.9), (4.10) and (4.11) to the right hand side of the inequality (4.7).

We shall take for

$$
\psi(x)=\prod_{i=1}^{n} \psi_{i}\left(\left|x_{i}\right|\right)
$$

where

$$
\psi_{i}(r)=\left\{\begin{array}{ccc}
1 & ; & 0 \leqq r \leqq k^{\prime} \rho \\
\frac{k \rho-r}{\left(k-k^{\prime}\right) \rho} ; & k^{\prime} \rho \leqq r \leqq k \rho \\
0 & ; & r \geqq k \rho
\end{array}\right.
$$

and denote

$$
V(t)=\int_{Q_{k_{p}}} v(x, t) \psi^{2} d x / \int_{Q_{k p}} \psi^{2} d x
$$

Then, we have

$$
\left.\int_{Q_{k \rho}} \psi^{2} v d x\right|_{t_{1}} ^{t_{2}} \geqq\left\{V\left(t_{2}\right)-V\left(t_{1}\right)\right\} \cdot\left|Q_{k^{\prime} \rho}\right|
$$

for the first term of the left hand side of (4.12), and

$$
\begin{aligned}
& \iint_{Q_{p} \times\left(t_{1}, t_{2}\right)} \psi^{2} v_{x}^{2} d x d t \\
& \geqq \frac{2}{k^{2}}\left(\frac{k^{\prime}}{k}\right)^{n} \cdot \frac{1}{\rho^{2}} \iint_{Q k_{\rho} \times\left(t_{1}, t_{2}\right)}\{v(x, t)-V(t)\}^{2} d x d t
\end{aligned}
$$

by applying Lemma 5 to the second term of the left hand side of (4.12), and furthermore we have 


$$
\begin{aligned}
& \iint_{Q_{k_{p} \times\left(t_{1}, t 2\right)}}\left\{k^{-2} \rho^{-2} \psi^{2}+\psi r_{\lambda}^{2}\right\} d x d t \\
& \leqq \frac{1}{\left(k-k^{\prime}\right)^{2}} \cdot\left(\frac{k}{k^{\prime}}\right)^{n} \cdot \frac{1}{\rho^{2}} \cdot\left|Q_{r^{\prime} \rho}\right|\left(t_{2}-t_{1}\right) .
\end{aligned}
$$

Since the right hand sides of both (4.13) and (4.14) remain unchanged if $v$ is replaced by $v+$ const, we normalize $v$ in $U$ by taking $V(0)=0$. Now we replace $v$ and $V$ by

$$
\begin{aligned}
& w(x, t)=v(x, t)-\bar{\gamma} \dot{t} \\
& W(t)=V(t)-\bar{\gamma} t,
\end{aligned}
$$

where

$$
\bar{\gamma}=\frac{\gamma}{\left(k-k^{\prime}\right)^{-}} \cdot\left(\frac{k}{k^{\prime}}\right)^{n} \cdot \frac{1}{\rho^{2}},
$$

then we obtain at last

$$
\begin{gathered}
\left\{W\left(t_{2}\right)-W\left(t_{1}\right)\right\}\left|Q_{k^{\prime} \rho}\right|+\frac{\gamma_{1}}{\rho^{2}} \iint_{Q_{k^{\prime} \rho \times\left(t_{1}, t_{2}\right)}}\{w(x, t) \\
-W(t)\}^{2} d x d t \leqq 0
\end{gathered}
$$

from (4.12), (4.13), (4.14), (4.15) and the definition (4.16), where

$$
\gamma_{1}=\frac{2}{k^{2}} \cdot\left(\frac{k^{\prime}}{k}\right) \text {. }
$$

Let $Q_{s}(t)$ be the set

$$
\left\{x \in Q_{k^{\prime} \rho} ; w(x, t)>s\right\} \subset Q_{k^{\prime} \rho}
$$

for $0<t<h_{1}^{\prime} \rho^{2}$. Note that

$$
w(x, t)-W(t)>s-W(t) \geqq s>0
$$

for any $s>0$ and $x \in Q_{s}(t)$ and $W(t)$ is monotone decreasing with $W(0)=0$. Then from (4.17) we have

$$
\begin{aligned}
\int_{t_{1}}^{t_{2}}\left|Q_{s}(t)\right| d t \leqq & \gamma_{1}^{-1} \rho^{2}\left|Q_{k^{\prime} \rho}\right|\left\{s-W\left(t^{\prime}\right)\right\}^{-2} \\
& \times\left[\left\{s-W\left(t_{2}\right)\right\}-\left\{s-W\left(t_{1}\right)\right\}\right]
\end{aligned}
$$

for some $t_{1}<t<t_{2}$ by the mean value theorem. Therefore we find

$$
\begin{gathered}
\int_{0}^{h_{1}^{\prime} \rho^{2}}\left|Q_{s}(t)\right| d t \leqq \gamma_{1}^{-1} \rho^{2}\left|Q_{k^{\prime} \rho}\right| \int_{0}^{h_{1}^{\prime} \rho^{2}}\{s-W(t)\}^{-2} d(s-W(t) \\
\leqq \gamma_{1}^{-1} \rho^{2}\left|Q_{k^{\prime} \rho}\right| s^{-1}
\end{gathered}
$$


and when we denote the measure of a set by $m\{\cdots \cdots\}$ and

$$
m(s)=m\left\{(x, t) \in U^{+} ; w(x, t)>s\right\},
$$

we obtain from (4.18)

$$
m(s) \leqq r_{1}^{-1} \rho^{2}\left|Q_{k^{\prime} \rho}\right| s^{-1} \text {. }
$$

From this estimate we can derive

$$
\iint_{U^{-} ; v>0} \sqrt{v} d x d t \leqq r\left|U^{+}\right|
$$

where $\gamma$ is a constant depending on $n, a, \lambda, k, k^{\prime}, h$ and $h_{1}^{\prime}$ alone. Replacing $t$ by $-t$ and $h_{1}^{\prime}$ by $-h_{2}^{\prime}$, we obtain from (4.19)

$$
\iint_{U^{-} ; v<0} \sqrt{-v} d x d t \leqq r \mid U^{-1}
$$

remembering the normalization $V(0)=0$. We have the result (4.2) from (4.19) and (4.20).

The Proof of Theorem 3. We set

$$
v=-\log \bar{u}, \bar{u}=|u|+L(\rho)
$$

for a supersolution $u$ and denote the measure;

$$
\begin{aligned}
\mu(s) & =M\left(U^{+}, U^{-}\right) \\
& =\frac{m\left\{x^{\prime} \in U^{+}, y^{\prime} \in U^{-}, v\left(x^{\prime}\right)-v\left(y^{\prime}\right)>s\right\}}{! U^{+} \| U^{-} \mid}
\end{aligned}
$$

for any $s>0$ and $U^{+}, U^{-} \subset U \subset R_{k \rho_{1}\left(k_{1}+h_{2}\right) \rho^{2}}$ defined at the beginning of this section. For a $\sigma>0$,

$$
\begin{aligned}
\mu(\sigma) \Phi(\sigma) & \leqq \int_{0}^{\sigma} \mu(s) d \Phi(s) \leqq \int_{0}^{\infty} \mu(s) d \Phi(s) \\
& \leqq \int_{0}^{\infty} \Phi(s) d(-\mu(s)) \leqq r
\end{aligned}
$$

with respect to the function $\Phi(\sigma)$ defined in (4.1) and the measure $\mu(s)$ defined in (4.21). The last inequality is true from (4.2) in Lemma 4. Then, we have

$$
\mu(s) \leqq \gamma \Phi(s)^{-1}
$$

for any $s>0$.

Now we shall define a family of rectangle subdomains;

$$
C_{\nu}=\left\{(x, t) ;\left|x_{i}\right|<k^{\prime} N^{-\nu} \rho,-h_{2}^{\prime} N^{-2 \nu} \rho^{2}<t<h_{1}^{\prime} N^{-2 \nu} \rho^{2}\right\}
$$




$$
\begin{aligned}
& C_{\nu}^{+}=\left\{(x, t) ;\left|x_{i}\right|<k^{\prime} N^{-\nu} \rho, h_{3}^{\prime} N^{-2 v} \rho^{2}<t<h_{1}^{\prime} N^{-2 \nu} \rho^{2}\right\} \\
& C_{\nu}^{-}=\left\{(x, t) ;\left|x_{i}\right|<k^{\prime} N^{-\nu} \rho,-h_{2}^{\prime} N^{-2 \nu} \rho^{2}<t<-h_{3}^{\prime} N^{-2 \nu} \rho^{2}\right\} \\
& C_{\nu}^{0}=\left\{(x, t) ;\left|x_{i}\right|<k^{\prime} N^{-\nu} \rho,-h_{3}^{\prime} N^{-2 \nu} \rho^{2}<t<h_{3}^{\prime} N^{-2 \nu} \rho^{2}\right\}
\end{aligned}
$$

for $\nu=0,1,2, \cdots$, where $N$ is an integer more than 2. From (4.22) we have

$$
M\left(C_{\nu}^{+}, C_{\nu}^{0}\right)+M\left(C_{\nu}^{0}, C_{\nu}^{-}\right) \leqq \gamma \cdot \Phi(s)^{-1}
$$

for any subdomain in the family (4.23), where $r$ is a constant depending on $n, a, \lambda, k, k^{\prime}, h_{1}^{\prime}, h_{2}^{\prime}$ and $h_{3}^{\prime}$ alone but independent of $\nu, \rho$ and $N$. The inequality (4.24) holds also for any rectangle subdomain transformed in parallel arbitrarily from that in the family (4.23), since the class of equation (1.1) is invariant under the parallel transformations of coordinates. While since the left hand side of the inequality (4.24) is the average of

$$
\frac{m\left\{x^{\prime} \in C_{\nu}^{+} ; v\left(x^{\prime}\right)-v\left(z^{\prime}\right)>s\right\}}{\left|C^{+}\right|}+\frac{m\left\{y^{\prime} \in C_{\nu}^{-} ; v\left(z^{\prime}\right)-v\left(y^{\prime}\right)>s\right\}}{\left|C^{-}\right|}
$$

over $z^{\prime} \in C_{\nu}^{0}$, there exists a constant $a_{\nu}$ for which

$$
\frac{m\left\{x^{\prime} \in C_{\nu}^{+} ; v\left(x^{\prime}\right)-a_{\nu}>s\right\}}{\left|C^{+}\right|}+\frac{m\left\{y^{\prime} \in C_{\nu}^{-} ; a_{\nu}-v\left(y^{\prime}\right)>s\right\}}{\left|C^{-}\right|}<\gamma \Phi(s)^{-1}
$$

Note that for any $\delta>0$, there exists an $s_{0}>0$ such that $\gamma \cdot \Phi\left(s_{0}\right)^{-1}$ $<\delta$. Then if we set

$$
f\left(x^{\prime}\right)=s_{0}^{-1} v\left(x^{\prime}\right), \phi_{\nu}=s_{0}^{-1} a_{\nu}
$$

we have

$$
\frac{m\left\{x^{\prime} \in C_{\nu}^{+} ; f\left(x^{\prime}\right)-\phi_{\nu}>1\right\}}{\left|C_{\nu}^{+}\right|}+\frac{m\left\{y^{\prime} \in C_{\nu}^{-} ; \phi_{\nu}-f\left(y^{\prime}\right)>1\right\}}{\left|C_{\nu}^{-}\right|}<\delta
$$

for $\nu=0,1,2, \cdots$.

Now we define

$$
p_{\nu}(s)=\frac{m\left\{x^{\prime} \in C_{\nu}^{+} ; f\left(x^{\prime}\right)-\phi_{\nu}>s\right\}}{\left|C_{\nu}^{+}\right|}+\frac{m\left\{y^{\prime} \in C_{\nu}^{-} ; \phi_{\nu}-f\left(y^{\prime}\right)>s\right\}}{\left|C_{\nu}^{-}\right|}
$$

with respect to domains of (4.23) and

$$
q(s)=\frac{m\left\{x^{\prime} \in D_{0}^{+} ; f\left(x^{\prime}\right)-\phi_{0}>s\right\}}{\left|D_{0}^{+}\right|}+\frac{m\left\{y^{\prime} \in D_{0}^{-} ; \phi_{0}-f\left(y^{\prime}\right)>s\right\}}{\left|D_{0}^{-}\right|}
$$

with respect to other subdomains; 


$$
\begin{aligned}
& D_{0}^{+}=\left\{(x, t) ;\left|x_{i}\right|<k^{\prime} \rho, h_{4}^{\prime} \rho^{2}<t<h_{1}^{\prime} \rho^{2}\right\} \\
& D_{0}^{-}=\left\{(x, t) ;\left|x_{i}\right|<k^{\prime} \rho,-h_{2}^{\prime} \rho^{2}<t<-h_{4}^{\prime} \rho^{2}\right\}
\end{aligned}
$$

for $0<h_{3}^{\prime}<h_{4}^{\prime}<h_{1}^{\prime}, h_{2}^{\prime}$. We can derive the following lemma;

Lemma 6. Suppose that

$$
p_{\nu}(1)<\delta, \nu=0,1,2, \cdots
$$

for some constant $\delta>0$ sufficiently small. Then we obtain

$$
q(s) \leqq a \cdot \exp (-b s)
$$

for any $s \geqq 1$, where $a$ and $b$ are constants depending on $n, \delta$ and $h_{1}^{\prime}$ through $h_{4}^{\prime}$ alone.

The proof of Lemma 6 can be seen in Appendix (see [6]). We shall continue our proof of Theorem 3. Since the assumption (4.28) is equal to (4.25), we can use the result (4.29) of Lemma 6. Taling for $\alpha=b / 2 s_{0}$, we have

$$
\begin{aligned}
& \frac{1}{\left|D_{0}^{+}\right|} \int_{D 0}+e^{\alpha\left(\imath\left(x^{\prime}\right)-a_{0}\right)} d x^{\prime}=\frac{1}{\left|D_{0}^{+}\right|} \int_{D 0^{+}} e^{b / 2\left(f\left(x^{\prime}\right)-\phi_{0}\right)} d x^{\prime} \\
= & \int_{-\infty}^{\infty} e^{b s / 2} d\left(-q^{+}(s)\right)=\int_{-\infty}^{\infty} q^{+}(s) d\left(e^{b s / 2}\right) \\
\leqq & r+\int_{1}^{\infty} a e^{-b s} d\left(e^{b s / 2}\right) \leqq r,
\end{aligned}
$$

where $q^{+}(s)$ in (4.30) denotes the first term of (4.26). In the same way we obtain

$$
\frac{1}{\left|D_{0}^{-}\right|} \int_{D 0} e^{\alpha\left(a_{0}-v\left(y^{\prime}\right)\right)} d y^{\prime} \leqq \gamma
$$

The inequalities (4.30) and (4.31) imply (4.3) of Theorem 3 since we can replace a parameter $h_{4}^{\prime}$ to $h_{3}^{\prime}$ and we have

$$
\iint_{C_{0}} \bar{u}^{\alpha} d x d t \cdot \iint_{C_{0}} \bar{u}^{-\alpha} d x d t \leqq \gamma\left|C_{0}^{+}\right|\left|C_{0}^{-}\right|
$$

by remembering the definition $v=-\log \bar{u}$.

\section{§5. The proof of Theorem 1}

We are now in a position to prove our main theorem (Theorem 1 ). For three subdomains (2.2) of $R$ we introduce new subdomains;

$$
C^{+}=\left\{(x, t) ;\left|x_{i}\right|<k^{\prime} \rho, i=1, \cdots, n, h_{4} \rho^{2}<t<h \rho^{2}\right\}
$$




$$
\begin{aligned}
& C^{-}=\left\{(x, t) ;\left|x_{i}\right|<k^{\prime} \rho, i=1, \cdots, n, \quad 0<t<h_{3} \rho^{2}\right\} \\
& R^{*}=\left\{(x, t) ;\left|x_{i}\right|<k^{*} \rho, i=1, \cdots, n, \quad 0<t<h_{2}^{-} \rho^{2}\right\}
\end{aligned}
$$

for $0<k_{1}<k^{*}<k^{\prime}<k$ and $0<h_{1}^{-}<h_{2}^{-}<h_{3}<h_{4}<h^{+}<h$. Assume that a given solution $u \geqq \delta>0$. Applying Theorem 3 to it with respect to $C^{+}$and $C^{-}$, we have from (4.3)

$$
M\left(\alpha, C^{-}\right) \leqq M\left(-\alpha, C^{+}\right)
$$

for some $\alpha>0$. Taking for $\delta=\alpha$ in Theorem 2, (3) with $R^{+} \subset C^{+}$, we have

$$
M\left(-\alpha, C^{+}\right) \leqq \gamma M\left(-\infty, R^{+}\right),
$$

and also for $\delta=\alpha$ in Theorem 2, (2) with $R^{*} \subset C^{-}$,

$$
M\left(p_{0}, R^{*}\right) \leqq \gamma M\left(\alpha, C^{-}\right)
$$

where $p_{0}$ is a constant more than 1 defined in Theorem 2, (2). Furthermore from Theorem 2, (1) with $R^{-} \subset R^{*}$, we obtain

$$
M\left(\infty, R^{-}\right) \leqq \gamma M\left(p_{0}, R^{*}\right) .
$$

Then using the four inequalities (5.1), (5.2), (5.3) and (5.4), we establish

$$
M\left(\infty, R^{-}\right) \leqq \gamma M\left(-\infty, R^{+}\right),
$$

where $\gamma$ is a constant which depends on $n, a, \lambda, k_{1}, h^{+}, h_{1}^{-}$and $h_{2}^{-}$alone. This inequality is independent of $\delta \rightarrow 0$. This proves Theorem 1 .

\section{Appendix}

We shall prove Lemma 6 in $\S 4$, which was extended from $F$. John and L. Nirenberg's Lemma [12] by J. Moser [5] [6]. Our proof is also essentially the same as his [6]. Remark that some constant $\delta>0$ is sufficiently small so as to satisíy three irequalities (A.3), (A.6) and (A.16) in the following paragraphs of our proof.

The Proof of Lemma 6. At first we shall subdivide $D_{0}^{+}$of (4.27) into $N^{n+2}$ congruent rectangle domains by subdividing the $t$ side into $N^{2}$ and the remaining sides into $N$ equal parts and denote them by $\left\{D_{1}^{+}\right\}$. In the $\nu$-th step, we obtain $N^{\nu(n+2)}$ congruent subrectangle domains denoted by $\left\{D_{\nu}^{+}\right\}$. Corresponding to the domain $D_{\circ}^{+}$ 
in (4.27), there exist three rectangle domains $C_{0}, C_{0}^{+}$and $C_{0}^{-}$in (4.23) and a constant $\phi_{0}$ in (4.25). Similarly we associate three rectangle subdomains $C_{\nu}, C_{\nu}^{+}$and $C_{\nu}^{-}$in (4.23) and a constant $\phi_{\nu}$ in (4.25) with each subdivided domains $D_{\nu}^{+}$for $\nu=1,2,3, \cdots$.

Proposition 1. (1) If an integer $N$ in (4.23) satisfies the inequality

$$
N^{2} \geqq \max \left\{\frac{h_{2}^{\prime}+h_{4}^{\prime}}{h_{4}^{\prime}-h_{3}^{\prime}}, 4\right\},
$$

then $D_{\nu}^{+} \subset D_{\nu-1}^{+}$implies $C_{\nu}^{-} \subset C_{\nu-1}^{+}$.

(2) If $D_{\nu}^{+} \subset D_{\nu-1}^{+}$then $\phi_{\nu}-\phi_{\nu-1} \leqq 2$.

The Proof of Proposition 1. (1) In order to obtain the result, it is sufficient that an inequality

$$
\frac{h_{2}^{\prime}+h_{4}^{\prime}}{N^{2}} \leqq h_{4}^{\prime}-h_{3}^{\prime}
$$

should be satisfied in comparing the height of the $t$ sides of rectangles $C_{\nu}^{-}$and $C_{\nu-1}^{+}$. This inequality (A.2) follows from the assumption (A. 1).

(2) If $\phi_{\nu}-\phi_{\nu-1}>2$, we would have

$$
\phi_{\nu}-f\left(x^{\prime}\right)>1 \text { or } f\left(x^{\prime}\right)-\phi_{\nu-1}>1
$$

for all $x^{\prime} \in C_{\nu}^{-} \subset C_{\nu-1}^{+}$. Therefore we have from the assumption (4.28) of Lemma 6 ,

$$
\begin{aligned}
\left|C_{\nu}^{-}\right| & \leqq m\left\{x^{\prime} \in C_{\nu}^{-} ; \phi_{\nu}-f\left(x^{\prime}\right)>1\right\} \\
& +m\left\{x^{\prime} \in C_{\nu-1}^{+} ; f\left(x^{\prime}\right)-\phi_{\nu-1}>1\right\} \\
& \leqq \delta\left|C_{\nu}^{-}\right|+\delta\left|C_{\nu-1}^{+}\right| \\
& =\delta\left[1+N^{n+2}\left(h_{1}^{\prime}-h_{3}^{\prime}\right) /\left(h_{2}^{\prime}-h_{3}^{\prime}\right)\right]\left|C_{\nu}^{-}\right|
\end{aligned}
$$

which is contradictory if the small constant $\delta>0$ satisfy

$$
\delta<\left\{1+N^{n+2}\left(h_{1}^{\prime}-h_{3}^{\prime}\right) /\left(h_{2}^{\prime}-h_{3}^{\prime}\right)\right\}^{-1} .
$$

Let $s_{0} \geqq 2$. We select from $\left\{D_{1}^{+}\right\}$domains for which $\phi_{1}-\phi_{0}>s_{0}$ and denote them by $\left\{\dot{D}_{1}^{+}\right\}$. The remaining rectangles are subdivided again. Similarly at the $\nu$-th step we select the rectangles $\left\{\dot{D}_{\nu}^{+}\right\}$for which $\phi_{\nu}-\phi_{0}>s_{0}$. 
We denote by $\dot{C}_{\nu}^{+}, \dot{C}_{\nu}^{-}, \dot{C}_{\nu}$ and $\dot{\phi}_{\nu}$ the rectangle domains and the constant which correspond to a selected domain $\dot{D}_{\nu}^{+}$. Let $\dot{D}=\cup \dot{C}_{\nu}^{+}$.

Proposition 2. (1) $s_{0}<\dot{\phi_{\nu}}-\phi_{0} \leqq s_{0}+2$.

(2) For $x^{\prime} \in D_{0}^{+}-\dot{D}$, we have $f\left(x^{\prime}\right)-\phi_{0} \leqq s_{0}+1$.

The Proof of Proposition 2. (1) The left inequality in (1) is clear. The right one is derived from

$$
\dot{\phi}_{\nu}-\phi_{\nu-1} \leqq 2 \text { and } \phi_{\nu-1}-\phi_{0} \leqq s_{0}
$$

since the first inequality follows from Proposition 1 (2) and the second one from the fact that $\dot{D}_{\nu}^{+}$has a rectangle $D_{\nu-1}^{+} \supset \dot{D}_{\nu}^{+}$which was not selected.

(2) For $x^{\prime} \in D_{0}^{+}-\dot{D}$ there exists a sequence of rectangles $\left\{D_{\nu}^{+}\right\}$ which were not selected and contain the point $x^{\prime}$, that is,

$$
\text { (A. 4) } \quad x^{\prime} \in D_{\nu}^{+} \subset C_{\nu}^{+}, \phi_{\nu}-\phi_{0} \leqq s_{0} \text {. }
$$

While there exists a point $x_{\nu}^{\prime} \in C_{\nu}^{+}$for every $D_{\nu}^{+}$such that

$$
f\left(x_{\nu}^{\prime}\right)-\phi_{\nu} \leqq 1
$$

since from the assumption (4.28) of Lemma 6,

$$
m\left\{x^{\prime} \in C_{\nu}^{+} ; f\left(x^{\prime}\right)-\phi_{\nu} \geqq 1\right\} \leqq \delta\left|C_{\nu}^{+}\right|<\left|C_{\nu}^{+}\right|
$$

if the small constant $\delta>0$ satisfies an inequality

$$
\text { (A. 6) } \delta<1 \text {. }
$$

Therefore from (A. 4) and (A. 5), we have

$$
f\left(x^{\prime}\right)-\phi_{0} \leqq\left|f\left(x^{\prime}\right)-f\left(x_{\nu}^{\prime}\right)\right|+1+s_{0}
$$

which implies the result of Proposition 2(2) since $f\left(x^{\prime}\right)$ is a continuous function in $R$.

Proposition 3. Denote the set

$$
\left\{x^{\prime} \in D_{0}^{+} ; f\left(x^{\prime}\right)-\phi_{0}>s_{0}-1\right\}
$$

by $S$. Then we have

$$
\text { (A. 7) } \quad|D| \leqq \gamma_{1}(1-2 \delta)^{-1}\left\{|S|+\gamma_{2} N^{-\left(s_{0}-1\right)}\left|D_{0}^{+}\right|\right\} \text {, }
$$

where

$$
\gamma_{1}=\frac{h_{1}^{\prime}+h_{2}^{\prime}}{h_{2}^{\prime}-h_{3}^{\prime}}+1, \gamma_{2}=\frac{h_{1}^{\prime}-h_{3}^{\prime}}{h_{1}^{\prime}-h_{4}^{\prime}} \cdot \frac{1}{N}
$$


The Proof of Proposition 3. From the assumption (4.28) of Lemma 6, we have

$$
\text { (A. 9) } \quad\left|\dot{C}_{\nu}^{-}\right| \leqq \frac{1}{1-2 \delta} m\left\{x^{\prime} \in C_{\nu}^{-} ; f\left(x^{\prime}\right)-\phi_{0}>s_{0}-1\right\}
$$

since $\phi_{\nu}-f\left(x^{\prime}\right) \leqq 1$ implies that

$$
f\left(x^{\prime}\right)-\phi_{0}=f\left(x^{\prime}\right)-\dot{\phi}_{\nu}+\dot{\phi}_{\nu}-\phi_{0}>-1+s_{0}
$$

Now, since the domains of $\left\{\dot{C}_{\nu}^{-}\right\}$are not always disjoint, we must select out of $\left\{\dot{C}_{\nu}^{-}\right\}$a subset of rectangle domains $\left\{\stackrel{*}{C}_{\lambda}^{-}\right\}$such that

$$
\left\{\begin{array}{l}
\left\{\mathscr{C}_{\lambda}^{*}\right\} \text { are not overlapping with one another } \\
\text { and each rectangle of }\left\{\dot{C}_{\nu}^{-}\right\} \text {always overlaps } \\
\text { with some rectangle of }\left\{\dot{C}_{\lambda}^{-}\right\}(\lambda \leqq \nu) .
\end{array}\right.
$$

The rectangles $\dot{D}_{\nu}^{+}$can be grouped into those for which the corresponding $\dot{C}_{\nu}^{-}$overlap with $\stackrel{C}{\bar{\lambda}}_{-}^{*}(\lambda \leqq \nu)$. These domains $\dot{D}_{\lambda}^{+}$are contained in a rectangle which lies above $\stackrel{*}{C}_{\lambda}^{-}$and has a height less than

$$
\gamma_{1}=\frac{h_{1}^{\prime}+h_{2}^{\prime}}{h_{2}^{\prime}-h_{3}^{\prime}}+1
$$

times the height of $\stackrel{*}{C}_{\lambda}^{-}$. Noting that both $\left\{\dot{D}_{\nu}^{+}\right\}$and $\left\{\stackrel{C}{C}_{\nu}^{-}\right\}$are nonoverlapping and making use of (A.9) we have

$$
\begin{aligned}
|\dot{D}| & =\Sigma\left|\dot{D}_{\nu}^{+}\right| \leqq r_{1} \Sigma\left|\stackrel{\leftrightarrow}{C}_{\lambda}^{-}\right| \\
& \leqq r_{1}(1-2 \delta)^{-1} \Sigma m\left\{x^{\prime} \in C_{\lambda}^{*} ; f\left(x^{\prime}\right)-\phi_{0}>s_{0}-1\right\} \\
& \leqq r_{1}(1-2 \delta)^{-1}\left\{|S|+\left|\left(C_{0}^{+}-D_{0}^{+}\right) \cap\left(\cup C_{\lambda}^{*}\right)\right|\right.
\end{aligned}
$$

While if we take an integer $\nu_{0}$ such that

$$
2 \nu_{0} \leqq s_{0}<2\left(\nu_{0}+1\right)
$$

for the fixed $s_{0} \geqq 2$, we have begun to select the rectangles of $\left\{\dot{D}_{\nu}^{+}\right\}$in fact from the $\nu_{0}$-th step of the decomposition since

$$
\phi_{\nu}-\phi_{0}=\left(\phi_{\nu}-\phi_{\nu-1}\right)+\cdots+\left(\phi_{1}-\phi_{0}\right) \leqq 2 \nu
$$

from the result of Proposition 1, (2). Therefore the domain $\left(C_{0}^{+}-D_{0}^{+}\right)$ $\cap\left(\cup C_{\lambda}^{*}\right)$ is contained in the union of $N^{\nu_{0}{ }^{n}}$ congruent rectangles $C_{\nu_{0}}^{+}$ which lie on the lowest position in $\left\{C_{\nu_{0}}^{+}\right\}$and hence 


$$
\begin{aligned}
& \left|\left(C_{0}^{+}-D_{0}^{+}\right) \cup\left(\cap \stackrel{*}{\lambda}_{\lambda}^{*}\right)\right| \leqq N^{\nu_{0} n}\left|C_{\nu_{0}^{+}}^{+}\right| \\
\leqq & \frac{N^{\nu_{0} n}}{N^{\nu_{0}(n+2)}}\left|C_{0}^{+}\right| \leqq \frac{1}{N^{s_{0}-2}} \cdot \frac{h_{1}-h_{3}}{h_{1}-h_{4}} \cdot\left|D_{0}^{+}\right| .
\end{aligned}
$$

From (A. 10) and (A. 11) we obtain the inequality (A.7) of Proposition 3.

Using the propositions above, we shall derive an estimate (4.28) of Lemma 6. Let $s_{0}=s+1$ for a $s \geqq 1$. Note that $f\left(x^{\prime}\right)-\phi_{0} \leqq s+2$ for $x^{\prime} \in D_{0}^{+}-\dot{D}$ from Propoistion $2(2)$ and $f\left(x^{\prime}\right)-\phi_{0}>s+4$ implies

$$
f\left(x^{\prime}\right)-\dot{\phi_{\nu}}=f\left(x^{\prime}\right)-\phi_{0}+\phi_{0}-\dot{\phi}_{\nu}>s+4-s-3=1
$$

for $x^{\prime} \in \dot{D}=\cup \dot{D}_{\nu}^{+}$. Then we obtain

$$
\begin{aligned}
& m\left\{x^{\prime} \in D_{0}^{+} ; f\left(x^{\prime}\right)-\phi_{0}>s+4\right\} \\
= & m\left\{x^{\prime} \in \dot{D} ; f\left(x^{\prime}\right)-\phi_{0}>s+4\right\} \\
\leqq & \Sigma m\left\{x^{\prime} \in \dot{D}_{\nu}^{+} ; f\left(x^{\prime}\right)-\phi_{\nu}>1\right\}
\end{aligned}
$$

and furthermore from the assumption (4.28) of Lemma 6 and the inequality (A.7) of Proposition 3 with $s_{0}=s+1$, we have

$$
\begin{aligned}
& \leqq \Sigma \delta\left|\dot{C}_{\nu}^{+}\right|=\left[\left(h_{1}^{\prime}-h_{3}^{\prime}\right) /\left(h_{1}^{\prime}-h_{4}^{\prime}\right)\right] \delta|\dot{D}| \\
& \leqq \frac{h_{1}^{\prime}-h_{3}^{\prime}}{h_{1}^{\prime}-h_{4}^{\prime}} \cdot \gamma_{1} \cdot \frac{\delta}{1-2 \delta}\left\{|S|+\frac{\gamma_{2}}{N^{s}}\left|D_{0}^{+}\right|\right\} .
\end{aligned}
$$

Then we obtain

$$
q^{+}(s+4) \leqq \gamma_{3} \delta(1-2 \delta)^{-1}\left\{q^{+}(s)+2 \gamma_{2} N^{-s}\right\},
$$

where we denote

$$
q^{+}(s)=\frac{m\left\{x^{\prime} \in D_{0}^{+} ; f\left(x^{\prime}\right)-\phi_{0}>s\right\}}{\left|D_{0}^{+}\right|}
$$

and

$$
\gamma_{3}=\frac{h_{1}^{\prime}-h_{3}^{\prime}}{h_{1}^{\prime}-h_{4}^{\prime}} \cdot \gamma_{1}
$$

with $\gamma_{1}$ and $\gamma_{2}$ in (A.8).

Making use of the notation $q(s)$ in (4.26), we can derive a recursion

$$
q(s+4) \leqq \gamma_{3} \delta(1-2 \delta)^{-1}\left\{q(s)+2 \gamma_{2} N^{-s}\right\}
$$


from (A. 12) and by the same arguments for the the rectangle $D_{0}^{-}$. Iteration of the recursion will yield the estimate (4.29) of Lemma 6 . For $1 \leqq s \leqq 4$, we have

$$
q(s) \leqq 2 \leqq 2 e^{4 b} e^{-b s},
$$

where $b>0$ is arbitrary. For $s>4$, setting $s=4 \sigma+s_{0}\left(\sigma\right.$; integer, $1 \leqq s_{0}$ $\leqq 4$ ) and using (A. 14), we have

$$
\begin{gathered}
q(s)=q\left(4 \sigma+s_{0}\right) \\
\leqq \gamma_{3} \delta(1-2 \delta)^{-1}\left\{q\left(4(\sigma-1)+s_{0}\right)+2 \gamma_{2} N^{-4(\sigma-1)}-s_{0}\right\} \\
\leqq\left(\frac{\delta_{3} \delta}{1-2 \delta}\right)^{\sigma}\left\{q\left(s_{0}\right)+\frac{2 \gamma_{2}}{N^{s_{0}}}\left[1+\left(\frac{N^{4} \gamma_{3} \delta}{1-2 \delta}\right)^{-1}+\cdots+\left(\frac{N^{4} \gamma_{3} \delta}{1-2 \delta}\right)^{-\sigma+1}\right]\right\} .
\end{gathered}
$$

Now we take for the small constant $\delta>0$ such that

$$
\frac{\gamma_{3} \delta}{1-2 \delta}<1 \text {, }
$$

that is,

$$
\text { (A. 16) } \quad \delta<\frac{1}{2+\gamma_{3}}=\left\{2+\frac{h_{1}^{\prime}-h_{3}^{\prime}}{h_{1}^{\prime}-h_{4}^{\prime}}\left[\frac{h_{1}^{\prime}+h_{2}^{\prime}}{h_{2}^{\prime}-h_{3}^{\prime}}+1\right]\right\}^{-1}
$$

from (A.13). For the fixed $\delta>0$ we enlarge $\gamma_{3}$ in the equality (A. 15) such that

$$
\frac{1}{N^{4}}<\frac{\gamma_{3} \delta}{1-2 \delta}<1,
$$

! if necessary. Then we obtain from (A. 15),

$$
\text { (A. 17) } \quad q(s) \leqq\left[\gamma_{3} \delta(1-2 \delta)^{-1}\right]^{\sigma} \cdot \gamma \text {, }
$$

where $\gamma$ is a constant depending on $\delta$ and $h_{1}^{\prime} \sim h_{4}^{\prime}$ alone. (A.17) implies

$$
q(s) \leqq a e^{-b\left(4 \sigma+s_{0}\right)}=a \cdot \exp (-b s),
$$

where we set

$$
\begin{aligned}
& a=\max \{r, 1\} e^{4 b} \\
& b=-4^{-1} \log \left[\gamma_{3} \delta(1-2 \delta)^{-1}\right] .
\end{aligned}
$$

This proves Lemma 6 .

\section{REFERENCES}

[1] Friedman, A., Partial differential equations of parabolic type, Prentice-Hall, En- 
glewood Cliffs, N. J., 1964.

[2] Moser, J, A new proof of de Giorgi's theorem concerning the regularity problem for elliptic differential equations, Comm. Pure Appl. Math. 13 (1960), 457-468.

[3] - On Harnack's theorem for elliptic differential equations, Ibid. 14 (1961), 577-591.

[4] - On the regularity problem for elliptic and parabolic differential equations, Partial Differential Equations and Continuum Mechanics, The University of Wisconsin Press (1961), 159-169.

[5] - A Harnack inequality for parabolic differential equations, Comm. Pure Appl. Math. 17 (1964), 101-134.

[6] Correction to "A Harnack inequality for parabolic differential equations”, Comm. Pure Appl. Math. 20 (1967), 231-236.

[7] Nash, J., Continuity of solutions of parabolic and elliptic equations, Amer. J. Math. 80 (1958), 931-954.

[8] Nirenberg, L, On elliptic partial differential equations, Ann. Scuola Norm. Sup. Pisa, 13 (1959), 1-48

[9] ґружков, С.Н., Априоры оценки дря обоьщенных решеппи̊ елпптических и параболических уравпенией второго порядка, Јокл. Акад. Наук СССР, 150 (1963), 748-751.

[10] — О некоторых своп̆ствах ретенпй еллиптическпх уравнепенцй, Локл. Акад. Наук СССР, 150 (1963), 470-473.

[11] - Априорные оценкп и некоторые свойстға репенці еллиптичскшх п параболпческих ураб̆нений, Мат. Сб. 65 (1964), 522-570.

[12] John, F. and L. Nirenberg, On functions of bounded mean oscillations, Comm. Pure Appl. Math. 14 (1964), 415-426.

[13] Serrin, J., Local behavior of solutions of quasi-linear equations, Acta Math. 111 (1964), 247-302.

[14] Kurihara, M., On a Harnack inequality for parabolic equations I, Funkcial. Ekvac. 17, No. 3 (1965), 3-57. (Japanese)

[15] - On a Harnack inequality for parabolic equations II, Ibid. 18, Nos. 1/2 (1965), 3-32. (Japanese)

Note added in proof (August 25, 1967):

Recently we knew that D.G. Aronson and J. Serrin and following them А. В. Иваиов had obtained the same results as ours.

Aronson, D. G. and J. Serrin, A Harnack inequality for nonlinear parabolic equations, Notices Amer. Math. Soc. 13, No. 3 (1966), 381.

Иванов, А.В., Неравенство гарнака для обобщенных решений кбазилинейных параболических уравнений второго порядка, Докл. Акад. Наук СССР, 173 (1967), 752-754.

Aronson, D.G. and J. Serrin, Local behavior of solutions of quasilinear parabolic equations, Arch. Rational Mech. Anal. Vol. 25, No. 2 (1967), 81-122. 
